\title{
Experimental Demonstration of a Novel QoS-Aware Hybrid Optical Network
}

\author{
Eduard Escalona $^{(1)}$, Yixuan Qin ${ }^{(1)}$, Georgios Zervas ${ }^{(1)}$, Joan Triay ${ }^{(2)}$, George Zarris ${ }^{(1)}$, \\ Norberto Amaya-Gonzalez ${ }^{(1)}$, Reza Nejabati ${ }^{(1)}$, Cristina Cervelló-Pastor ${ }^{(2)}$, Dimitra Simeonidou ${ }^{(1)}$ \\ (1) High-Performance Networks Group, CSEE Department, University of Essex, eescal@essex.ac.uk \\ (2) Department of Telematics Engineering, Universitat Politecnica de Catalunya (UPC)
}

\begin{abstract}
This paper presents a hybrid optical test-bed controlled by a hardware-accelerated control plane capable of dynamically provisioning the most suitable route according to the requirements of the client technology and the physical characteristics of the network.
\end{abstract}

\section{Introduction}

Over the last few years, a noticeable amount of effort has been devoted to the study of methods for mitigating the effect of physical layer impairments over the Quality of Transmission (QoT) of optical lightpaths. Most of the proposed impairment mitigation solutions require complex routing algorithms that try to accommodate each requested lightpath with specific requirements. However, they do not offer any flexibility to the user application to dynamically adjust the quality of their transmission and allocated bandwidth based on their needs.

The work carried out in this paper shows a novel approach for the integration of a hybrid multi-technology, multi-bitrate optical network with a hardware-accelerated dynamic impairment aware provisioning system that can facilitate on demand user-controlled QoT adjustment. The presented mechanism, besides finding the best possible route, it allows to dynamically adjust the transmission rate, giving the application a degree of flexibility by reducing the provided bandwidth instead of directly blocking a request when QoS conditions are not met. This approach is particularly useful for applications that can work at multiple rates such as video streaming.

The adopted solution has been implemented and evaluated in a real hybrid test-bed with actual physical impairment monitoring and a hardware-accelerated QoT estimator.

\section{Test-bed architecture}

One of the key issues in dynamic optical networks is the control plane. An additional step to the traditional GMPLS control plane procedures has been introduced to consider the application service class. An overlay control plane model allows the user to invocate a connection request using an interface (UNI) directly connected to an edge node. The architecture scenario proposed in this paper is shown in Fig. 1. It deploys a UNI gateway which implements a Connection Web Service towards the client and the standard UNI interface towards the GMPLS core network. The UNI gateway also interacts with a QoT estimator prior to signal the connection establishment.

The test-bed reported in this paper comprises a client side, a GMPLS-controlled core network and a server side. The client connects to the server using different transport technologies at different transmission rates $(2.5 \mathrm{~Gb} / \mathrm{s}, 10 \mathrm{~Gb} / \mathrm{s}, 40 \mathrm{~Gb} / \mathrm{s})$. Each of these transport clients is configured at GMPLS as TELinks with a specific Transport Network Address (TNA) and bandwidth capacity; this information is then propagated by the routing protocol. When the client requires a network connection to the server it sends the request to the UNI gateway using the Connection Web Service.

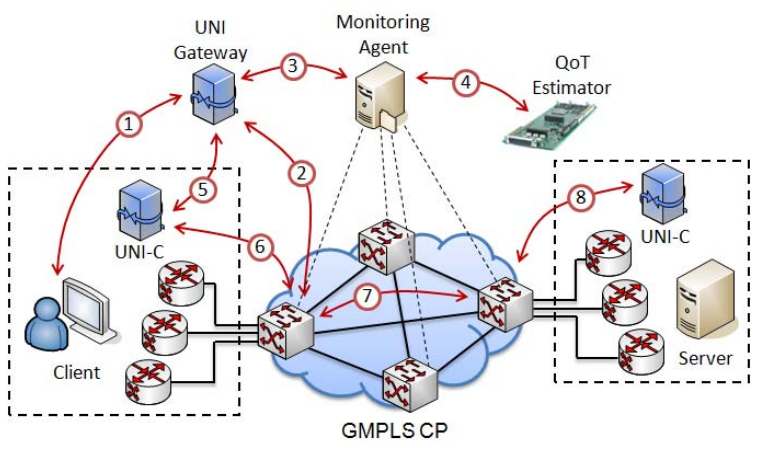

Fig. 1: Test-bed architecture

In a typical optical connection request, a user specifies source, destination and bandwidth. With the implemented Connection Web Service the user also indicates the service class that will use the connection. It can be either: Real Time, Streaming or Transactional. This will be used at the UNI gateway to determine the expected QoS by mapping the acceptable Packet Loss Rate (PLR) per application into QoT (Q Factor) values according to Tab. 1.

Tab. 1: QoS for Service class

\begin{tabular}{|c|c|c|c|}
\hline & PLR & BER & Q \\
\hline Real Time & $<5 \mathrm{E}-5$ & $<1.1 \mathrm{E}-6$ & $\sim 13.4$ \\
\hline Streaming & $<1 \mathrm{E}-3$ & $<2.8 \mathrm{E}-5$ & $\sim 12.0$ \\
\hline Transactional & $<1 \mathrm{E}-2$ & $<2.3 \mathrm{E}-4$ & $\sim 10.9$ \\
\hline
\end{tabular}


The PLR classification and the Q Factor relation with respect to the Bit Error Rate (BER) have been taken from the literature ${ }^{1,2}$, while the correlation between packet loss rate and BER has been experimentally obtained by monitoring continuous data traffic with variable packet size. It is of great importance to fulfil these requirements to guarantee the QoS to the user application. For instance, video quality is directly affected by packet loss, above all in real time applications such as HD videoconferencing.

Upon a connection request from the user (Fig. 1 - Step 1) the UNI gateway interacts with the Path Computation Engine (PCE) to obtain a route (Step 2). At the same time it gathers from GMPLS the list of the already established LSPs that share any link with the calculated route and sends the information, including the desired transmission rate, to the Monitoring Agent (MA) (Step 3). The MA adds physical layer parameters obtained through actual monitoring and preconfigured static values that characterize each link, to form a complete picture of the network. This information is transmitted to the hardware-accelerated QoT estimator, which calculates the impact of the new LSP over the existing LSPs in the form of $Q$ factor values (Step 4). These values are sent back to the UNI gateway, which accepts or rejects the route according to the previously specified service class and requested QoS. If the route is found appropriate, standard GMPLS mechanisms are triggered to establish the connection (Steps 58). Otherwise, a disjoint route is calculated by the PCE and the QoT is estimated again. If a disjoint acceptable route is not possible, the UNI gateway retries Step (3) with the next possible transmission rate specified by the client. If no possible combination rate/route is found the connection request is blocked.

\section{Experimental Evaluation}

In order to experimentally evaluate the feasibility of the proposed architecture a multi-bitrate testbed has been implemented as depicted in Fig. 2. The core network is comprised of four Fiber Switch Capable (FSC) Calient Optical CrossConnects (OXC) forming a mesh network. The link between OXC1 and OXC3 is a $110 \mathrm{~km}$ dispersion-compensated dark fibre used to recreate a Lambda Switch Capable (LSC) link between the two nodes and to show the effect of new established wavelengths over the others under limit conditions. The client is connected to OXC1 by three possible transmission clients $(2.5 \mathrm{~Gb} / \mathrm{s}, 10 \mathrm{~Gb} / \mathrm{s}$ and $40 \mathrm{~Gb} / \mathrm{s})$. The server is comprised of three Ultra High Definition video streaming servers with $2 \mathrm{~K}, 4 \mathrm{~K}$ and $8 \mathrm{~K}$ resolution which, in our case, require a bit rate of $1.2 \mathrm{Gbps}$, $7.64 \mathrm{Gbps}$ and $24 \mathrm{Gbps}$ respectively. Each of them is connected to OXC3 by three transmission clients $(2.5 \mathrm{~Gb} / \mathrm{s}, 10 \mathrm{~Gb} / \mathrm{s}$ and $40 \mathrm{~Gb} / \mathrm{s}$ ). With this scenario, when a new connection request arrives, standard mechanisms would simply choose the shortest available path, but the QoS provided may not be enough for the user application. In turn, the proposed approach checks if the QoS is met and retries a different transmission rate instead.

As a proof of concept three initial scenarios have been tested with an incoming connection request from 10.10.10.x to 20.20.20.x with three possible clients (40G, $10 \mathrm{G}$ and $2.5 \mathrm{G}$ ) and a streaming service class. Tab. 2 shows the iteration results for each tested scenario. In the first scenario, no connection exists in the network. The first path calculation attempt returns route OXC1-OXC3 and Q Factor 15.60. According to Tab. 1 the connection is accepted and the $8 \mathrm{~K}$ video server is used on the $40 \mathrm{~Gb} / \mathrm{s}$ channel.

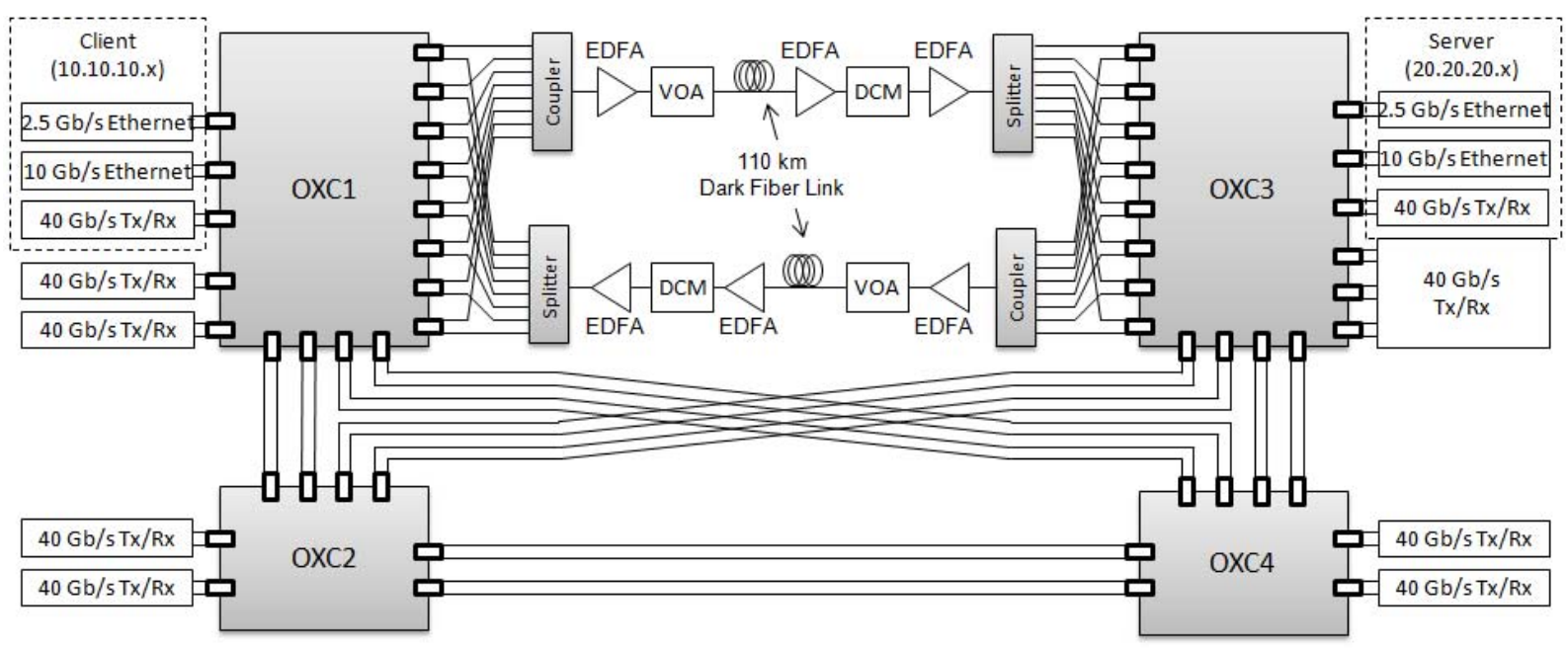

Fig. 2: Test-bed data plane 
Tab. 2: Connection request example

\begin{tabular}{|c|c|c|c|c|}
\hline \multicolumn{5}{|c|}{ Scenario 1 (No existing LSPs) } \\
\hline 1 & OXC1-OXC3 & $40 G$ & $Q=15.60$ & $\checkmark$ \\
\hline \multicolumn{5}{|c|}{ Scenario 2 (4 existing LSPs) } \\
\hline 1 & OXC1-OXC3 & $40 G$ & $Q=11.52$ & $x$ \\
\hline 2 & OXC1-OXC2-OXC3 & $40 G$ & $Q=15.95$ & $\sqrt{ }$ \\
\hline \multicolumn{5}{|c|}{ Scenario 3 (5 existing LSPs) } \\
\hline 1 & OXC1-OXC3 & $40 G$ & $Q=11.52$ & $x$ \\
\hline 2 & No route & $40 G$ & ---- & $x$ \\
\hline 3 & OXC1-OXC3 & $10 G$ & $Q=15.74$ & $\checkmark$ \\
\hline
\end{tabular}

In the second scenario the following $40 \mathrm{~Gb} / \mathrm{s}$ lightpaths have been pre-established as LSP1: OXC1-OXC3, LSP2: OXC2-OXC1-OXC3, LSP3: OXC4-OXC1-OXC3 and LSP4: OXC4-OXC1OXC3. Now, with the same request as in the previous scenario, the first route attempt (OXC1OXC3) returns an estimated $Q$ Factor of 11.52 due to the existence of the other lightpaths in the same link. Thus, the route is rejected and a disjoint one is calculated (OXC1-OXC2-OXC3) with $Q$ Factor 15.95, which is accepted.

Finally, the last scenario considers an extra $40 \mathrm{~Gb} / \mathrm{s}$ connection, LSP5: OXC1-OXC2-OXC3. In this case, the first attempt (OXC1-OXC3, Q Factor $=11.52$ ) does not meet the required QoS and the second attempt is unable to find a disjoint route due to lack of resources. Then, the UNI gateway retries with the $10 \mathrm{~Gb} / \mathrm{s}$ channel for route OXC1-OXC3. This time, the returned $Q$ Factor is 15.74 because of the change in the transmission rate, so the route is accepted and the path allocated, allowing the streaming of the $4 \mathrm{~K}$ video. It is important to note that the estimated $Q$ Factor values obtained depend on the specific characteristics and actual state of the physical layer.

\section{Hardware Acceleration}

The main drawback of the presented approach is that the QOT estimation carries out very complex calculations which increase exponentially with the number of lightpaths. This leads to a high execution time in the order of tens or hundreds of seconds, limiting the scalability of the network.

In order to address this problem, an FPGAbased hardware-accelerated QoT estimator is used $^{3}$. It dramatically decreases the processing time making the setup delay suitable for dynamic and real-time environments. The main benefit of using an FPGA-based hardwareaccelerated tool is to enable high-speed execution of timing critical processes in QoT which need fast response and low delay. In this experiment, the QoT has been integrated within the FPGA fabric and its embedded processor.

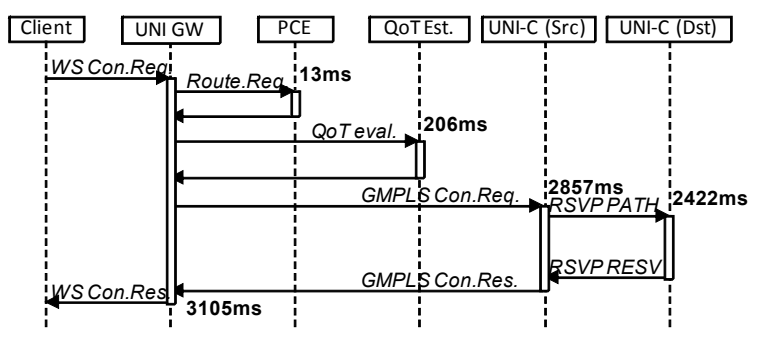

Fig. 3: Timeline diagram

Fig. 3 illustrates the workflow diagram of a connection request and setup. It shows that the hardware acceleration achieves a QoT estimation in the order of 200 milliseconds and further experimental results prove that increasing the number of lightpaths to 100 keeps the QoT processing time below 1 second. The overall end-to-end setup delay is around 3 seconds, mainly due to the GMPLS signalling, which includes the call signalling, the connection signalling, the OXC configuration time and verification of the cross-connections.

\section{Conclusions}

This paper presents the experimental demonstration of an impairment aware control plane solution based on standard GMPLS for a hybrid multi-technology, multi-bitrate network. The ability to make real time routing decisions considering different transmission bitrates based on QoT estimations upon real monitored parameters increase the granularity of the network and open the possibility of taking into account user and applications requirements. This adds a degree of flexibility by allowing a dynamic reduction in the transmission rate when introducing new LSPs at higher rates degrades the Quality of Transmission. The obtained results demonstrate that the proposed architecture with a hardware-accelerated QoT estimator is suitable for dynamic real environments.

\section{Acknowledgements}

The work presented in this paper has been supported by GÉANT3 project as well as DICONET project funded by the European Commission through the $7^{\text {th }}$ ICT-Framework program.

\section{References}

1 A. Stavdas, Core and Metro Networks, Wiley (2010)

$2 \mathrm{P}$. Pavon, Offline Impaiment Aware RWA Algorithms for Cross-Layer Planning of Optical Networks, JLT vol.27, no.12, June (2009)

3 Y. Qin et al., Hardware Accelerated Impairment Aware Control Plane, OFC (2010) 